\title{
Madelung deformity with Vickers ligament
}

\author{
Hee Kyung Kim
}

Received: 25 February 2009 /Accepted: 13 April 2009/Published online: 20 May 2009

(C) Springer-Verlag 2009

An 11-year-old girl with chronic left wrist deformity and pain underwent MRI evaluation. Coronal intermediateweighted sequence with fat suppression showed a triangular deformity with medial tilt of the distal radius and proximal migration of the triangular-shaped lunate (Fig. 1). The triangular fibrocartilage complex (TFCC) demonstrated diffuse thickening and oblique orientation (Fig. 1, arrow). These findings are suggestive of Madelung deformity. Sagittal intermediate-weighted sequence with fat suppression showed diffuse thickening of the radiolunate ligament, suggesting Vickers ligament (Fig. 2).

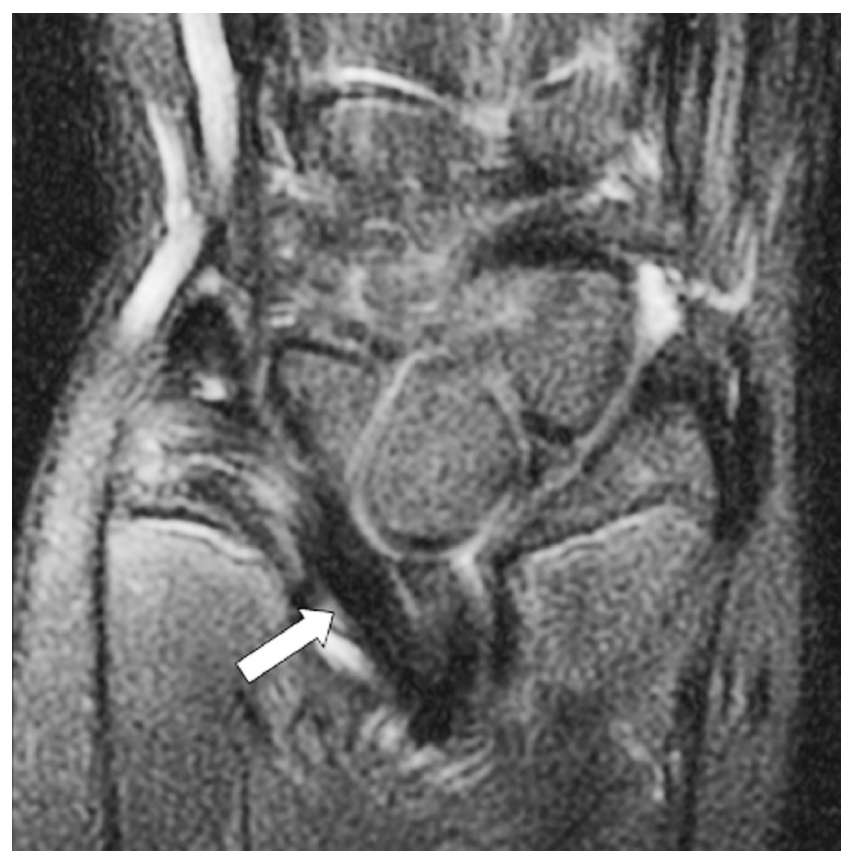

Fig. 1 Coronal image

\section{H. K. Kim $(\bowtie)$}

Department of Radiology, Cincinnati Children's Medical Center, 3333 Burnet Ave.,

Cincinnati, OH 45229, USA

e-mail: Hee.Kim@cchmc.org

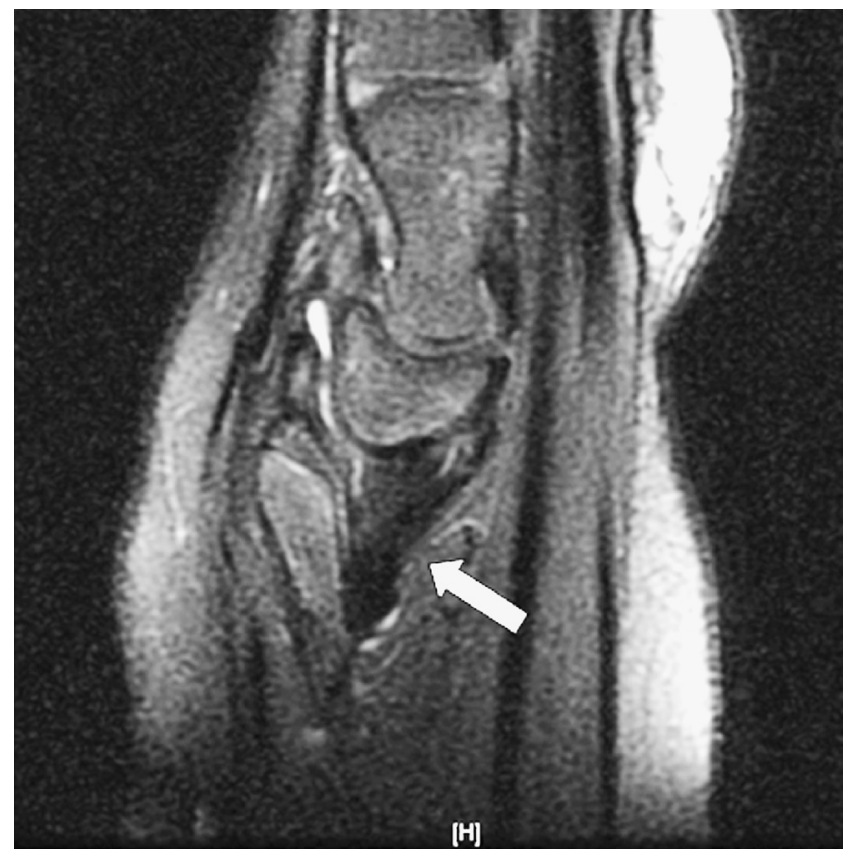

Fig. 2 Sagittal image

Madelung deformity is a developmental growth disturbance of the distal radius caused by focal dysplasia of the physis with triangular deformity and medial tilt of the distal radius. It is associated with a thickened radiolunate ligament, Vickers ligament, which tethers the lunate and the TFCC to the deformed distal radius [1]. Corrective osteotomy of the radius and release of the thickened radiolunate ligament is performed to treat the deformity and to achieve symptomatic improvement [2].

\section{References}

1. Stoller DW, Li AE, Lichtman DM et al (2007) The wrist and hand. In: Stoller DW (ed) Magnetic resonance imaging in orthopaedics and sports medicine. Lippincott Williams Wilkins, Philadelphia, pp 1627-1846

2. Harley BJ, Brown C, Cummings K et al (2006) Volar ligament release and distal radius dome osteotomy for correction of Madelung's deformity. J Hand Surg [Am] 31:1499-1506 